\title{
Strong family history and cigarette smoking as risk factors of coronary artery disease in young adults $\star$
}

\author{
JAMES H CHESEBRO, VALENTIN FUSTER, LILA R ELVEBACK, \\ ROBERT L FRYE \\ From the Division of Cardiovascular Diseases and Internal Medicine and the Department of Medical Research \\ Statistics and Epidemiology, Mayo Clinic and Mayo Foundation, Rochester, Minnesota, USA.
}

SUMMARY This study is based on 435 consecutive patients age 50 or less who had coronarye arteriography. There were 335 patients with coronary artery disease and 100 with normal coronaryarteries. Risk factors reviewed were "packet-years" of cigarette smoking, family history of coronary ${ }_{\infty}$ disease in first-degree relatives 50 years of age or less, age- and sex-corrected serum cholesterol ando triglycerides, hypertension, and diabetes. By univariate analysis, each risk factor except hypertension and diabetes was significantly more frequent in patients with coronary disease than in those without. By multivariate analysis of all risk factors in patients with and without coronary disease, the? male or female patient with coronary disease could best be identified by the strong family history, cigarette smoking history, and age- and sex-corrected serum cholesterol. The percentage of patients with coronary disease when the three risk factors were present was $95 \%$, two factors present $88 \%, \infty$ one factor present $67 \%$, none of the three $25 \%$, strong family history alone $90 \%$, smoking alone $66 \%$, and serum cholesterol $>80$ th centile alone $52 \%$.

Coronary atherosclerotic disease starts at a young age and takes many years to progress into the symptomatic stage. ${ }^{1}$ By the time the first symptom appears, the disease is advanced to two- or three-vessel involvement in most patients, ${ }^{2}$ and indeed sudden death may be the first symptom in nearly one-third of patients. ${ }^{3}$ It is imperative that these patients be identified at a young age before the first symptom appears, for it is at the early stages of the disease, rather than in the later symptomatic stages, that intervention to prevent progression of disease and death is most likely to be successful. Furthermore, when the disease appears at a young age, it might be more rapidly progressive unless interventions are made. Improvement in identification of the large majority of young persons in the general population at high risk of developing coronary disease by noninvasive methods is needed. As a first step in approaching this problem we have reviewed the risk factors in 435 patients 50 years of age or younger who had coronary arteriography.

\section{Patients and methods}

Patients selected for study included consecutive Caucasian patients of 50 years of age or less who had

*This investigation was supported in part by a research grant from the National Institute of Health, Public Health Service.

Accepted for publication 17 June 1981 coronary arteriography because of typical or atypical $\stackrel{\mathbb{\Omega}}{\rightarrow}$ chest pain from 1 July 1975 to 30 June 1977. Theo patients were divided into two groups: (1) $335_{\triangle}^{\Im}$ patients with coronary artery disease (median and mean ages 46 and 45 years respectively, $88 \%$ men) who had at least one coronary artery stenosed $>50 \%$ ind luminal diameter $\left(138\right.$ or $41 \%$ had previous myocar- $-\frac{7}{-}$ diai infarction) and (2) 100 patients (median and mean ages 44 and 43 years respectively, $58 \%$ men) who had. angiographically normal coronary arteries, no previous myocardial infarction by history or electro- cardiographic criteria, and no clinical evidence of? peripheral arteriosclerosis obliterans as judged by normal arterial pulses and absence of claudication and bruits. Patients with coronary lesions $<50 \%$ in luminald diameter, and associated valvular, congenital, or cardiomyopathic heart disease were excluded.

\section{RISK FACTORS}

The following risk factors were considered.

\section{(1) Cigarette smoking}

The number of "packet-years" (number of packs $(20$ cigarettes per pack) per day times number of yearsक्षे smoked) of smoking was determined from the patien? as previously described and along with the currento smoking status (smoking within a week before coro웅 
nary arteriography). In the text we will use number of years of smoking to mean the numbers of packet-years of smoking.

\section{(2) Family history}

We only considered a strong family history of coronary disease (myocardial infarction, sudden death, exertional angina, or disease documented by coronary angiography) in first-degree relatives who were age 50 years and under as previously described. ${ }^{4}$

\section{(3) Hyperlipidaemia (at the time of admission for coro- nary arteriography)}

Hypercholesterolaemia and hypertriglyceridaemia were analysed as previously described ${ }^{4}$ and defined as at or above the 95 th or 80 th centile ${ }^{\star}$ for a patient's age and sex, and for discriminant function analysis as the age- and sex-corrected serum cholesterol and triglyceride relative deviate.

\section{(4) Systemic hypertension}

This was taken as positive if the diagnosis $(>160 / 95$ $\mathrm{mmHg}$ ) had been made before coronary arteriography (and patients were susequently given antihypertensive medication) or if at the evaluation for coronary arteriography the blood pressure was $\geqslant 160 / 95 \mathrm{mmHg}$ on three occasions.
(5) Diabetes mellitus

This was defined as two fasting plasma glucose values $\geqslant 6.6 \mathrm{mmol} / \mathrm{l}(\geqslant 120 \mathrm{mg} / \mathrm{dl})$ normal $\leqslant 5.5 \mathrm{mmol} / \mathrm{l}$ (normal $\leqslant 100 \mathrm{mg} / \mathrm{dl}$ ) or insulin dependence.

\section{CORONARY ARTERIOGRAPHY}

Selective coronary cineangiography was performed with and without cranial angulation and as previously described..$^{5}$ Patients with a maximal coronary artery stenosis $<50 \%$ (22 patients) were excluded from the study to make the division between coronary disease and normal coronary arteries as definite as possible.

\section{STATISTICS}

Significance of univariate analysis of risk factors was tested by Student's t test or $\chi^{2}$. Stepwise linear discriminate function analysis was carried out according to the RMD P7M computer program of the Health Sciences Computing Facility, University of California at Los Angeles.

\section{Results}

PREVALENCE OF RISK FACTORS

The Figure shows the percentage of patients with each risk factor for those with and without coronary artery disease. Each risk factor except hypertension and

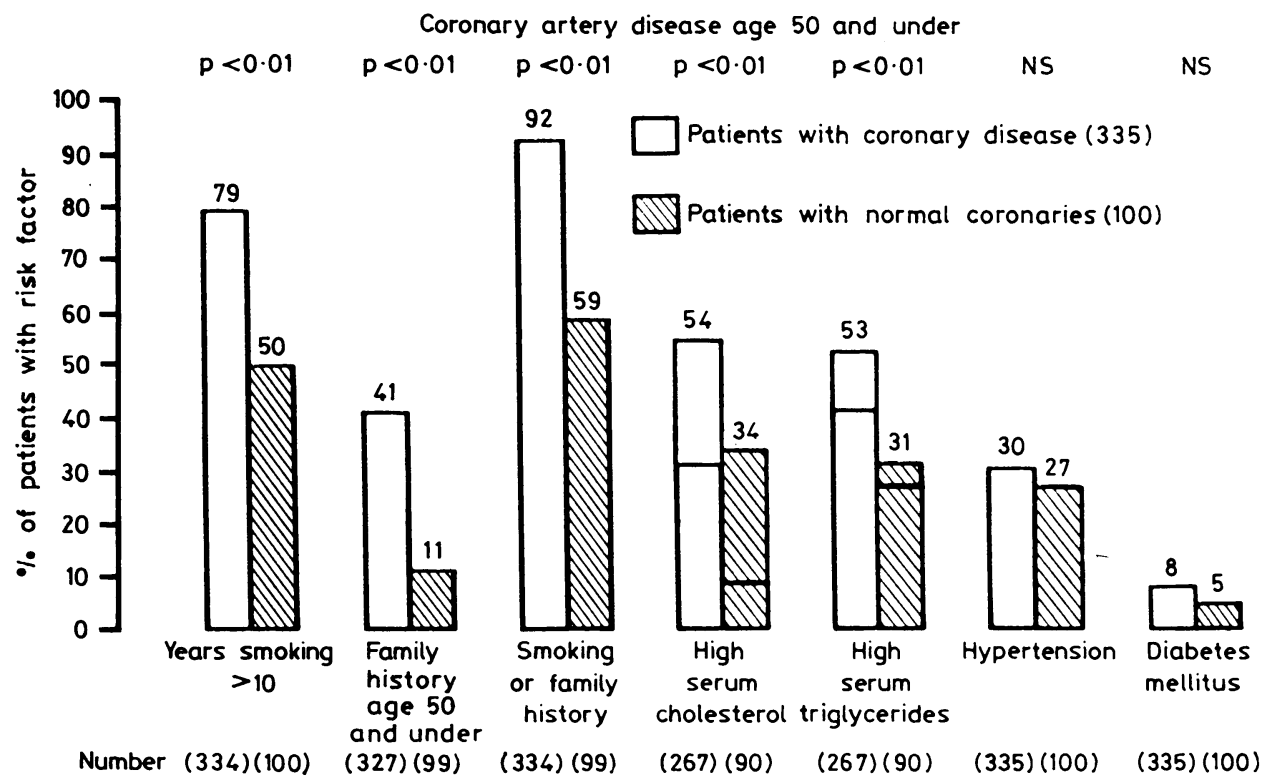

Fig. Univariate analysis of risk factors in young patients with coronary artery disease and those with normal coronary arteries. Serum cholesterol and serum triglycerides were taken at $\geqslant 80$ th centile for age and sex (top of bar) and 95 th centile (solid line in bar). Not shown is current smoking status for coronary disease (26\%) and normal coronary (22\%) patients. Each risk factor except diabetes, hypertension, and current smoking status was significantly $(p<0.01)$ more frequent in patients with coronary disease than in those without. Number of patients in each risk factor subgroup is shown below each bar. Patients in whom a risk factor was unknown were excluded from analysis: smoking 1 , family history 8 , cholesterol and triglycerides 68 (coronary disease), and 10 (normal).

*Age-sex specific centiles were established by our laboratory on the basis of 458 carefully screened healthy adults. At age 45 the 80 th centiles (in mg/ $100 \mathrm{ml}$ ) for cholesterol were 250 for men and 235 for women, and the 95 th centiles were 285 in men and 267 in women. For triglycerides the 95 th centiles at age 45 were 195 in men and 125 in women. 
diabetes mellitus was present in a significantly $(p<0.01)$ higher percentage in the patients with coronary artery disease. Cigarette smoking was the single most frequent risk factor in patients with coronary disease, being present in $79 \%$ of the patients. Family history of coronary artery disease at a young age was found in $41 \%$ of patients with coronary artery disease and only $11 \%$ of normal patients. A cholesterol level at or above the 95th centile was found in $31 \%$ of patients with coronary disease and $9 \%$ of patients with normal coronary arteries. A triglyceride level at or above the 95 th centile was found in $41 \%$ of patients with coronary disease but also in $27 \%$ of normal patients. We also looked at the percentage of patients with cholesterol and triglyceride levels at or above the 50th and the 80th centiles for age and sex (Fig. and Table 1). The presence of a history of cigarette smoking or a positive family history (or both) was found in $92 \%$ of patients with coronary disease and $59 \%$ of patients with normal coronary arteries (Fig.).

Table 1 Risk factor prevalence in patients with and without coronary disease at different cut points in three of the continuous variables: the patients are those in whom all risk factors were available for analysis.

\begin{tabular}{lllllll}
\hline & Males & \multicolumn{3}{c}{ Females } & Total \\
& CAD & No CAD & CAD & No CAD CAD & No CAD \\
n=351 & 232 & 53 & 30 & 36 & 262 & 89 \\
\hline & $\%$ & $\%$ & $\%$ & $\%$ & $\%$ & $\%$ \\
Age $<40$ & 16 & 40 & 10 & 17 & 15 & 27 \\
Family history & 41 & 11 & 37 & 8 & 40 & 10 \\
Packet-years & & & & & & \\
$\quad 0$ & 16 & 21 & 20 & 69 & 17 & 40 \\
$\geqslant 10$ & 79 & 68 & 73 & 28 & 79 & 52 \\
$\geqslant 30$ & 49 & 32 & 40 & 8 & 48 & 22 \\
Cholesterol & & & & & & \\
$>$ P $_{50}$ & 81 & 58 & 87 & 58 & 82 & 58 \\
$>$ P $_{80}$ & 53 & 34 & 60 & 33 & 54 & 34 \\
$<$ P $_{95}$ & 28 & 6 & 47 & 11 & 30 & 8 \\
Triglyceride & & & & & & \\
$>$ P $_{50}$ & 86 & 66 & 77 & 83 & 85 & 73 \\
$>$ P $_{80}$ & 51 & 30 & 70 & 33 & 53 & 31 \\
$>$ P $_{95}$ & 39 & 25 & 57 & 31 & 41 & 27 \\
Hypertensive & 30 & 40 & 10 & 17 & 28 & 30 \\
\hline
\end{tabular}

PREDICTIVE VALUE OF RISK FACTORS

Linear discriminant analysis

Stepwise linear discriminant function analysis in the 351 patients in whom all the risk factors were available for analysis (family history, number of packetyears of cigarette smoking, current smoking status, age- and sex-corrected cholesterol and triglyceride levels, hypertension, systolic blood pressure, diastolic blood pressure, and diabetes mellitus) showed that the young patient with coronary artery disease could best be identified by cigarette smoking, family his-
Table 2 Stepwise linear discrimination between those with aftd without coronary disease

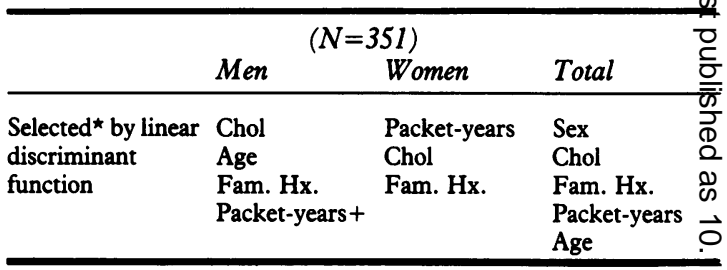

$\mathrm{N}$, number of patients.

$\star$ Using $F$ to enter $\geqslant 4(p<0.05)$ when age is dropped.

Fam. Hx., strong family history; Packet-years, number of packet years of cigarette smoking; Chol, age- and sex-corrected serum cholesterol relative deviate.

tory, cholesterol, sex, and age. Table 2 summarise the results of stepwise linear discrimination between those with and those without coronary artery disease for men, women, and the total group and lists the variables selected in order of their discriminating power. Sex-corrected cholesterol, strong family hisర్ tory, and packet-years of cigarette smoking weeg selected by linear discriminant function in both sexes Age was not selected in women probably because of the smaller age spread (see Table 1).

\section{Univariate analysis}

Table 3 describes the coronary disease prevalence eight subgroups defined in terms of strong fami $\vec{F}$ history, cholesterol level above the 80th centile for age and sex, and 10 years or more of cigarette smoking the 351 patients ( 262 with and 89 without coronary disease) for whom data on all three of these risk factors were available. When none of these risk factors are present $25 \%$ of patients have coronary disease, with one of these factors $67 \%$ have coronary disease, with two of these factors $88 \%$ have disease, and the increase is small ( $88 \%$ to $95 \%$ ) with a third risk factop.

Table 3 Coronary disease prevalence in subgroups defined family history, cigarette smoking, and cholesterol risk factors

\begin{tabular}{|c|c|c|c|c|}
\hline $\begin{array}{l}\text { Family } \\
\text { history }\end{array}$ & $\begin{array}{l}\text { Cholesterol } \\
>\mathrm{P}_{80}\end{array}$ & $\geqslant 10$ & $\mathrm{~N}$ & \% CAA \\
\hline $\begin{array}{l}+ \\
+ \\
+ \\
- \\
+ \\
- \\
-\end{array}$ & $\begin{array}{l}+ \\
- \\
+ \\
+ \\
- \\
- \\
+ \\
-\end{array}$ & $\begin{array}{l}+ \\
+ \\
- \\
+ \\
- \\
+ \\
- \\
-\end{array}$ & $\begin{array}{l}42 \\
32 \\
19 \\
83 \\
21 \\
95 \\
27 \\
32 \\
-351\end{array}$ & 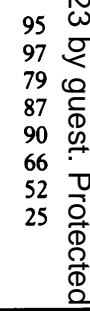 \\
\hline
\end{tabular}

$\mathrm{N}$, number of patients; CAD, coronary artery disease. 
Strong family history is the single risk factor that is associated with the greatest prevalence of coronary disease $(90 \%)$, followed by $\geqslant 10$ years cigarette smoking $(66 \%)$, and cholesterol level above the 80 th centile (52\%). These above results would change if the cut points for centile of cholesterol and number of years of cigarette smoking were changed. Table 1 describes the results in the same 351 patients included in Table 3 without regard to subgroup analysis but at different cut points for centile cholesterol and triglycerides and number of years of smoking.

\section{Discussion}

This study clearly shows the association between the strong family history, cigarette smoking, and age- and sex-corrected cholesterol risk factors and the presence of coronary disease in young patients having coronary angiography. These are factors of recognised importance in the development of coronary artery disease in the general population, but the strong family history risk factor (in first degree relatives at age 50 and under) is not widely recognised and has not frequently been looked for in large prospective epidemiological studies. While it is not surprising that the association penetrates to this angiographic subgroup, the strength of the association may have been materially altered by the selection process of patients for coronary angiography. A safe, noninvasive measure of the degree of coronary stenosis comparable in accuracy to angiography could determine the strength of this association in the general population, but such a noninvasive method is not yet available.

Studies aimed at risk factor prediction of the presence or absence of coronary disease which involve individuals who are candidates for coronary arteriography have the advantage of eliminating the misclassification which results from errors of clinical diagnosis. ${ }^{6}$ Individuals who have had coronary arteriography, however, do not necessarily represent the general population since, on the one hand, those with coronary disease and no symptoms of coronary disease are underrepresented, and those who die at the initial event are missing. Though the subgroup with normal coronary arteries is missing those with no symptoms, this subgroup has been shown, when restudied after 12 to 104 months, to have a benign prognosis $^{7}$ and normal coronary arteries. ${ }^{8}{ }^{9}$ This group was also free of clinical peripheral atherosclerotic disease.

It is difficult to establish the precise order of importance of the three major risk factors. For a single risk factor, strong family history is associated with the largest increase in coronary disease prevalence, and years of smoking is next (Table 3). By stepwise linear discrimination all three are significant in identifying the coronary patient, but cholesterol ranks ahead of the other two in men and in the total group while years of smoking ranks first in women (Table 2). In univariate analysis the three risk factors are all highly significant. For example, using strong family history, the difference in the percent positive gives $\chi_{1}^{2}=27$, cigarette smoking $\geqslant 30$ years gives $\chi_{1}^{2}=18$, and cholesterol above the 90th centile for age and sex also gives $\chi_{1}^{2}=18$. The method of statistical observation and the cutoff point used for a continuous variable may change the order of apparent importance. We found from practical and clinical standpoints that it is uncommon to find a patient 50 years of age or younger with angiographically proven coronary artery disease who does not have a cigarette smoking history or a positive family history of coronary artery disease at a young age. It is also uncommon to find angiographically normal coronary arteries in a young person who has both of the latter risk factors or all three of these risk factors $(\leqslant 5 \%)$ as noted in Table 3.

Cigarette smoking is a well-recognised primary risk factor $^{10}{ }^{11}$ in men ${ }^{12}$ and women ${ }^{13}$ and a recently recognised cause of secondary complications in patients with known coronary artery disease who continue to smoke, compared with former smokers or nonsmokers. ${ }^{11}{ }^{13} \mathrm{We}$ found that a history of 10 years or more of cigarette smoking increases the risk of having coronary artery disease whether or not the patient is currently smoking. Thus, in taking the history it is important to determine a quantitative estimate of previous cigarette smoking since only a small number of patients may be currently smoking. We observed only $26 \%$ of patients with coronary disease and $22 \%$ of patients with normal coronary arteries to be currently smoking. The mechanism whereby smoking contributes to the development and complications of coronary artery disease is unknown but is discussed by us elsewhere. ${ }^{4}$ Unfortunately, there is an alarming trend toward increased smoking in women and young people, ${ }^{14}$ and, therefore, better methods leading to prevention and cessation of smoking are needed.

It is important to note the strength of the family history risk factor in a patient's history since the occurrence at a young age appears to be associated with coronary disease in other young family members who present with symptoms of possible coronary disease. It is of interest that clinical manifestations of coronary disease show similarities within sibships such as cardiac deaths being most common in sibs of the fatal infarction probands. ${ }^{15}$ The younger the age at diagnosis the greater the risk of premature coronary disease in other first-degree relatives. ${ }^{16}$ Slack and Evans ${ }^{17}$ found that the risk of coronary death under age 55 is 5.2 times that of the general population if a male first-degree relative has died from coronary 
disease under age 55 , and 2.8 times if the relative is a women under age 65 . These increases in risk are about twice that experienced by middle-aged men with serum cholesterol over $7.8 \mathrm{mmol} /(300 \mathrm{mg} / \mathrm{dl})$ or diastolic blood pressure over $105 \mathrm{mmHg} .{ }^{18}$ Since the strong family history risk factor is dependent on sibship size and birth order of the subject in relation to sibs, the absence of sibs or the subject being oldest among sibs, would probably lead to understimation of the frequency of this risk factor. The genetic factor that contributes to the development and progression of coronary artery disease is unknown, can only be speculated upon, and is discussed by us elsewhere. ${ }^{4}$ Unlike studies from the Finnish population ${ }^{19}$ we found that those with and without a positive family history did not differ significantly in any of the other risk factors. We found the cholesterol and triglyceride distributions for patients with a positive and those with a negative family history of coronary artery disease were not significantly different: In addition, in the subgroup of patients with both strong family history and cigarette smoking regardless of serum cholesterol, $96 \%(71 / 74)$ had coronary artery disease. It is also striking that $90 \%$ of patients with the strong family history risk factor alone had coronary disease. Asymptomatic young adults with the strong family and/or the cigarette smoking risk factors have a high incidence of a shortened ${ }^{51} \mathrm{Cr}$ platelet survival half-life suggesting enhanced platelet-arterial wall interaction ${ }^{4}$ and the possibility that these individuals with risk factors and/or shortened ${ }^{51} \mathrm{Cr}$ platelet survival half-life may have subclinical atherosclerotic disease and might be good candidates for an early intervention trial in the "prevention" of coronary artery disease.

Hypercholesterolaemia is a well-documented primary risk factor ${ }^{1011}$ and a recently documented secondary risk factor ${ }^{20}$ of coronary artery disease. Lowering of serum cholesterol may retard angiographic progression of coronary artery disease ${ }^{21}$ and reduce mortality from coronary artery disease. ${ }^{22}$ The increase in coronary artery disease with increasing serum lipid concentrations holds over the entire range, without any critical serum level to separate risk from non-risk groups. ${ }^{23}$ The association was stronger with cholesterol than with triglyceride. Premature or accelerated atherosclerosis occurs in patients who have hyperlipoproteinaemia, but hyperlipoproteinaemia, as we also observed, is neither necessary nor sufficient for atherosclerosis. ${ }^{24}$ Because the incidence of coronary artery disease and the levels of serum cholesterol in presumably healthy persons in the United States are higher than those in most countries of the world (including countries where smoking is prevalent), cholesterol may play a permissive role in the development of atherosclerosis. For example, in Japan, where smoking is very prevalent, the levels of serum choles- terol and the incidence of coronary artery disease afe very low. ${ }^{25}$

Hypertension is both a primary ${ }^{10}$ and a secondary risk factor. In the two groups of young patiengs having angiography, we found no difference in the proportion with hypertension. It is possible that if had been able to integrate the level of increase ove time, an association might have been found. In addro tion, some of those with the longest duration of higt levels of blood pressure might have been lost by death at the intitial manifestation of coronary artery disease (sudden death or fatal myocardial infarction).

Although these risk factors identify groups at high risk for having coronary artery disease at a young age, they are not as specific as coronary arteriography for identification of coronary disease. Not all risk factors? however, have necessarily been identified. Tle angiographic groups offer an opportunity for intensive studies to identify new risk factors. Since platelets appear to play a role in the development of atherosclerotic disease, we evaluated the relation between the risk factors found here and platelet con sumption as assessed by ${ }^{51} \mathrm{Cr}$ platelet survival half life. ${ }^{4}$ We are also following medically treated coronary disease patients in a prospective, randomised, double blind, platelet inhibitor drug trial, and will be able see if the strong family history risk factor or platele survival half-life are secondary risk factors in the angiographic progression of coronary artery disease.

Thus, the strong family history is an independe $\overrightarrow{B t}$ risk factor not associated with a clustering of other risk factors. It helps identify young patients at risk having coronary disease along with the cigaret. smoking history and serum cholesterol and appears $\overline{\mathrm{D}}$ be associated with enhanced platelet-arterial wal interaction. ${ }^{4}$

\section{References}

1 McGill HC Jr. The geographic pathology of atherosclerosis? Baltimore: William \& Wilkins, 1968.

2 Fuster V, Frye RL, Connolly DC, Danielson MA Elveback LR, Kurland LT. Arteriographic patterng early in the onset of the coronary syndromes. $\mathrm{Br}$ Heart 1975; 37: 1250-5.

3 Spain DM, Bradess VA. Sudden death from corona heart disease; survival time, frequency of thrombi, and cigarette smoking. Chest 1970: 58: 107-10.

4 Fuster. V, Chesebro JH, Frye RL, Elveback LR. Platelet survival and the development of coronary artery disease in the young adult: the effects of cigarette smoking strong family history and medical therapy. Circulation 1981; 63: 546-51.

5 Chesebro JH, Ritman EL, Frye RL, et al. Videometîn analysis of regional left ventricular function before apd after aortocoronary artery bypass surgery: correlation of peak rate of myocardial wall thickening with late pos 
operative graft flows. $\mathcal{F}$ Clin Invest 1976; 58: 1339-47.

6 Welch CC, Proudfit WL, Sheldon WC. Coronary arteriographic findings in 1000 women under age 50. Am f Cardiol 1975; 35: 211-5.

7 Kemp HG Jr, Vokonas PS, Cohn PF, Gorlin R. The anginal syndrome associated with normal coronary arteriograms: report of a six-year experience. $A m \mathcal{F} \mathrm{Med}$ 1973; 54: 735-42.

8 Gensini GG, Kelly AE. Incidence and progression of coronary artery disease: an angiographic correlation in 1,263 patients. Arch Intern Med 1972; 129: 814-27.

9 Marchandise B, Bourassa MG. Prognostic significance of normal coronary arteries and mild coronary atherosclerosis (abstract). Am f Cardiol 1977; 39: 328.

10 Wilhelmsen L, Wedel H, Tibblin G. Multivariate analysis of risk factors for coronary heart disease. Circulation 1973; 48: 950-8.

11 Moss AJ. Profile of high risk in people known to have coronary heart disease: a review. Circulation 1975; 52, suppl 3: 147-54.

12 Doll $R$, Peto $R$. Mortality in relation to smoking: 20 years' observations on male British doctors. $\mathrm{Br}$ Med $\mathcal{F}$ 1976; ii: 1525-36.

13 Sloane D, Shapiro S, Rosenberg L, et al. Relation of cigarette smoking to myocardial infarction in young women. $N$ Engl f Med 1978; 298: 1273-6.

14 Hanley JA, Robinson JC. Cigarette smoking and the young: a national survey. Can Med Assoc $\mathcal{F}$ 1976; 114: 511-7.

15 Rissanen AM. Familial aggregation of coronary heart disease in a high incidence area (North Karelia, Finland). Br Heart f 1979; 42: 294-303.

16 Rissanen AM. Familial occurrence of coronary heart disease: effect of age at diagnosis. Am $\mathcal{F}$ Cardiol 1979; 44: 60-6.

17 Slack J, Evans KA. The increased risk of death from ischaemic heart disease in first degree relatives of 121 men and 96 women with ischaemic heart disease. $f$ Med Genet 1966; 3: 239-57.
18 AMA Inter-Society Commission for heart disease resources Atherosclerosis Study Group and Epidemiology Study Group. Primary prevention of the atherosclerotic diseases. Circulation 1970; 42: A-55-95.

19 Rissanen AM, Nikkilä EA. Aggregation of coronary risk factors in families of men with fatal and non-fatal coronary heart disease. Br Heart $\mathcal{F} 1979 ;$ 42: 373-80.

20 Coronary Drug Project Research Group. Natural history of myocardial infarction in the Coronary Drug Project: long-term prognostic importance of serum lipid levels. Am f Cardiol 1978; 42: 489-98.

21 Nikkilä EA, Viikinkosi P, Valle M. Effect of lipid lowering treatment on progression of coronary atherosclerosis assessed by angiography (abstract). Circulation 1978; 58, suppl 2:50.

22 Miettinen M, Turpeinen O, Karvonen MJ, Elosuo R, Paavilainen E. Effect of cholesterol-lowering diet on mortality from coronary heart disease and other causes: a twelve-year clinical trial in men and women. Lancet 1972; ii: 835-8.

23 Gotto AM, Gorry GA, Thompson JR, et al. Relationship between plasma lipid concentrations and coronary artery disease in 496 patients. Circulation 1977; 56: 875-83.

24 Roberts WC, Ferrans VJ, Levy RI, Fredrickson DS. Cardiovascular pathology in hyperlipoproteinemia: anatomic observations in 42 necropsy patients with normal or abnormal serum lipoprotein patterns. Am $\mathfrak{F}$ Cardiol 1973; 31: 557-70.

25 Keys A, Blackburn $\mathrm{H}$. Background of the patient with coronary heart disease. Prog Cardiovasc Dis 1963; 6: $14-44$.

26 Passamani ER. Summary of ongoing clinical trials of platelet-active drugs in cardiovascular disease. Circulation 1980; 62, suppl 5: 106-10.

Requests for reprints to Dr James H Chesebro, Mayo Clinic, Rochester, Minnesota 55901, USA. 